\title{
Bite force and masticatory efficiency in individuals with different oral rehabilitations
}

\author{
Laner B. Rosa ${ }^{1}$, Cesar Bataglion ${ }^{2}$, Selma Siéssere ${ }^{1}$, Marcelo Palinkas ${ }^{2}$, Wilson Mestriner Júnior ${ }^{3}$, \\ Osvaldo de Freitas ${ }^{4}$, Moara de Rossi ${ }^{1}$, Lígia Franco de Oliveira ${ }^{1}$, Simone C. H. Regalo ${ }^{1^{*}}$ \\ ${ }^{1}$ Department of Morphology, Stomatology, and Physiology, School of Dentistry, University of São Paulo, Ribeirão Preto, Brazil \\ ${ }^{2}$ Department of Dentistry, School of Dentistry, University of São Paulo, Ribeirão Preto, Brazil \\ ${ }^{3}$ Department of Pediatric Clinics, Preventive and Community Dentistry, Ribeirão Preto Dental School, University of São Paulo, \\ Ribeirão Preto, Brazil \\ ${ }^{4}$ Department of Pharmaceutical Sciences, Ribeirão Preto School of Pharmaceutical Sciences, University of São Paulo, Ribeirão Preto, \\ Brazil \\ Email: schregalo@forp.usp.br
}

Received 7 December 2011; revised 14 January 2012; accepted 7 February 2012

\begin{abstract}
Objective: This study was analyzed adult individuals rehabilitated with different types of dentures, with the purpose of verifying the effect that different types of denture rehabilitation have on maximal bite force and masticatory efficiency. The aim of this study is to facilitate diagnosis and prognosis, bringing contributions to the quality and stability of treatments Materials and Methods: Fifty individuals were divided into five groups: one control group with ten dentate individuals and another four groups formed according to the type of rehabilitation treatment. Maximal bite force was recorded on the first molar regions, and masticatory efficiency rates were recorded on the right, left and habitual sides. Results: Data related to the maximal bite force recordings were statistically significant across the analyzed groups [ANOVA for $p$ $\leq$ 0.01]. The data related to masticatory efficiency rates also showed statistical significance across all groups [ANOVA for $p \leq 0.05$ ] in the three tested conditions [mastication on the right, left and habitual sides]. Conclusion: The group of individuals rehabilitated with implants and single crowns showed greater bite force values and masticatory efficiency rates compared to the other groups, and the treatment with implant-supported mandibular overdenture improved the function compared to conventional complete dentures, thus evincing that the stomatognathic system has different functional behaviors depending on the type of oral rehabilitation.
\end{abstract}

Keywords: Bite Force; Masticatory Performance; Oral Rehabilitation

\footnotetext{
${ }^{*}$ Corresponding author.
}

\section{INTRODUCTION}

Across the world, numerous individuals have been affected with dental loss, including both young, between their 15 and 19 years, and elderly individuals, which cause physiological, neuromuscular and functional disorders. To correct these disorders, rehabilitation treatments with adequate dentures, either fixed or removable, are indicated. This implies that dentists must have the necessary knowledge and skills to rehabilitate the stomatognathic system, recovering functions, esthetics and the social and psychological wellbeing of patients who need oral rehabilitation [1].

The number of teeth in the oral cavity and the lack of dental contact are important factors that affect the action of the masticatory system [2]. Several factors determine the final outcome of mastication and the teeth are key factors in this process, principally the posterior teeth, where food is fragmented. This fragmentation depends on the total area of the occlusal surface and, therefore, on the number of teeth in occlusion [3]. Maintaining a reasonable number of healthy natural teeth is the best way to guarantee good masticatory efficiency [4].

Bite force is an important variable to investigate oral function related to occlusal factor, dentition, dentures, treatment with implants, orthognatic surgery, temporomandibular disorders and neuromuscular changes [5]. Muscle force and the number of functional teeth are determinant factors in masticatory. Measuring maximum bite force is an attempt to quantify the force that mandible elevator muscles can make [3].

In this view, the objective of this study was to investigate the association between masticatory efficiency and maximum bite force in individuals rehabilitated with implants, complete dentures, partial dentures, overdentures and natural dentitions. 


\section{MATERIALS AND METHODS}

\subsection{Subjects}

Participants were recruited from an outpatient universitybased oral rehabilitation clinic at Ribeirão Preto School of Dentistry and at a particular dentistry office. Written informed consent was obtained for each patient according to the Research Ethics Committee (Process: 2008.1. 167.58.3) and in accordance with resolution 196/96 of the Brazilian National Health Council.

The selection of the sample and the inclusion/exclusion criteria were determined through anamnesis and clinical exams. The anamnesis interview provided information on personal data, medical records, dental history, presence of parafunctional habits, and possible symptoms of temporomandibular dysfunction (RDC-Research Diagnostic Criteria).

Inclusion criteria were: 1) individuals who had been rehabilitated with at least 10 single-crowns over implants; 2) complete dentures with satisfactory stability and retention and intact alveolar mucosa and ridge; 3) removable partial dentures replacing at least ten missing teeth; 4) fully dentate individuals, with normal occlusion and no signs or symptoms for temporomandibular disorder (RDC); 5) age between 35 and 70 years.

The items used as exclusion criteria were the presence of local or systemic-originated disorders which may impair the craniofacial growth or the masticatory system, such as neurological disorders, cerebral palsy; use of medications that can interfere directly or indirectly on muscular activity, such as antihistamines, sedatives, cough syrups, homeopathic remedies or other drugs with a depressive action on the Central Nervous System; treatments that may interfere on muscular activity, directly or indirectly, during the period of the study, such as orthodontic treatments, phonoaudiological therapy, and otorhinolaryngologic treatment and the use of dentures considered clinically unsatisfactory.

Among 300 patients evaluated, 95 were found to be eligible for the research and 50 were enrolled and then divided into five groups. Group I consisted of 10 individuals rehabilitated with at least 10 implants and single crowns; Group II had 10 individuals rehabilitated with upper complete dentures and implant-supported overdentures on the lower arch; Group III consisted of 10 rehabilitated with complete dentures on the upper and lower arches; Group IV consisted of 10 individuals rehabilitated with removable partial dentures on the upper and lower arches, and Group V comprised by 10 dentate individuals (control group).

For the present study, the individuals who comprised the study groups had been rehabilitated at least six months earlier, were pleased with their prosthetic rehabilitations and reported having adequate masticatory ef- ficiency.

\subsection{Maximal Bite Force}

Bite force records were taken with a digital dynamometer, model IDDK (Kratos, Cotia, São Paulo, Brazil), with a $1000 \mathrm{~N}$ capacity, adapted to the mouth. The apparatus has a "set-zero" key, which allows the exact control of the values obtained and also "peak" registers, that facilitates the record of the maximal force during measures. It has two arms with plastic disks on each end, over which the force to be measured is applied. Its high-precision charge cell and electronic indicator force circuit, supply precise measures easily viewed on a digital display. The dynamometer was cleaned with alcohol and disposable latex finger cots (Wariper-São Paulo-Brazil) were positioned on the biting arms as a biosecurity measure. The participants were given detailed instructions and bite tests were performed before the actual recordings were made, in order to ensure the reliability of the procedure. The volunteers were then asked to bite the dynamometer three times with maximal force, with a two-minute rest interval between records. Evaluations were performed in the first molar (left and right). Maximal bite force was measured in N through the "peak" force record indicated on the screen for subsequent analysis. The highest value out of three records was considered as the individual's maximal bite force [1].

\subsection{Masticatory Efficiency Test}

For the masticatory efficiency test, beads obtained by ionotropic gellification of an aqueous dispersion of $2 \%$ pectin containing $50 \%$ solids and fuchsin in a $1.0 \mathrm{M}$ calcium chloride solution were used. After preparation, the beads were coated with a 5\% Eudragit solution (Eudragit E100) in a solvent mixture of $10 \%$ acetone in absolute ethanol. Next, $250 \mathrm{mg}$ of the beads were packed in polyvinyl acetate capsules with $0.67 \mathrm{~mm}$ thick walls, inner diameter of $7.6 \mathrm{~mm}$ and outer diameter of $8.95 \mathrm{~mm}$, and sealed. The subjects were instructed to chew the beads in their free habitual manner. The test was stopped after 20 seconds and the beads were collected into a container identified with subject and test number. Mastication tests on the right and left were later carried out following the same procedures. After mastication, the content of the capsule was dissolved in $5 \mathrm{ml}$ of water by mixing constantly for 30 seconds. The solution was then filtered through qualitative filter paper and the extracted dye was quantitated in nanometers (nm) with a Beckman DU-7 UV-Visible Spectrophotometer (Beckman Inc., Palo Alto, CA, USA). This permitted the determination of masticatory efficiency on the basis of the concentration of extracted fuchsin. The analyses were carried out at the Pharmacotechnique Laboratory of the Faculty of Phar- 
maceutical Sciences of Ribeirão Preto, University of São Paulo [6].

\section{RESULTS}

The researcher that performed the statistic analysis was blind to the group distribution. One way ANOVA and Duncan post-hoc test were used to investigate significant differences among the groups. The association between masticatory efficiency and the maximal bite force was verified using Pearson's Bivariate Correlation. SPSS 17.0 software was used and a level of significance of 5\% was considered. The data obtained for masticatory efficiency showed a statistically significant difference between all groups, in the three tested conditions (mastication on the right side, left side and habitual). The highest efficiency indexes were found in the groups of individuals rehabilitated with implant and single crowns and in dentate individuals (control group). The group of individuals rehabilitated with upper and lower removable partial dentures presented a masticatory efficiency index similar to that of the group rehabilitated with upper complete denture and mandible implant-supported overdenture. The group rehabilitated with upper and lower complete denture showed the lowest masticatory efficiency index (Table 1).

The data concerning maximum bite force measurements were statistically significant between the analyzed groups, with the highest force values found in the control group and the group with individuals rehabilitated with single crowns and implants presented, both for the right and left molars. The group rehabilitated with upper and lower partial removable dentures presented intermediate values, while the smallest values were found in the group with upper complete dentures and lower overdenture, and in the group with upper and lower complete denture (Table 2). Similar values were found for the right and left molar region, hence the comparison between sides showed no statistical significance.

The correlation analysis between bite force and masticatory efficiency was performed independently for the left and right molar. The analysis of bite force between groups showed a positive correlation for the right molar region for habitual mastication and mastication on the right and left sides, with data significant for $p<0.05$. A positive correlation was found for the left molar region in all mastication tests, with significant data observed only for habitual mastication (Table 3).

\section{DISCUSSION}

The oral condition of most people is characterized by complete or partial edentulism, which causes extensive changes to one's health, such as a reduced masticatory capacity, digestive, nutritional, speaking, hearing and
Table 1. Mean values and standard error of masticatory efficiency indexes in the different groups, during the mastication of beads on the right, left and habitual sides (ANOVA).

\begin{tabular}{lccc}
\hline \multicolumn{1}{c}{ Groups and Mastication } & N & $\begin{array}{c}\text { Means and } \\
\text { Standard } \\
\text { Deviation }\end{array}$ & Significance \\
\hline Habitual Mastication & & & S \\
Implants and Single Crowns & 10 & $1.53 \pm 0.25$ & \\
Upper CD and Lower Overdenture & 10 & $1.15 \pm 0.09$ & \\
Upper and Lower CD & 10 & $0.75 \pm 0.03$ & \\
Upper and Lower PRD & 10 & $1.14 \pm 0.08$ & \\
Control & 10 & $1.41 \pm 0.09$ & \\
Mastication on Right Side & & & \\
Implants and Single Crowns & 10 & $1.63 \pm 0.35$ & \\
Upper CD and Lower Overdenture & 10 & $1.18 \pm 0.09$ \\
Upper and Lower CD & 10 & $0.73 \pm 0.05$ & \\
Upper and Lower PRD & 10 & $1.12 \pm 0.12$ & \\
Control & 10 & $1.35 \pm 0.06$ & \\
Mastication on Left Side & & & \\
Implants and Single Crowns & 10 & $1.57 \pm 0.30$ \\
Upper CD and Lower Overdenture & 10 & $1.12 \pm 0.10$ \\
Upper and Lower CD & 10 & $0.76 \pm 0.03$ \\
Upper and Lower PRD & 10 & $1.04 \pm 0.07$ & \\
Control & 10 & $1.33 \pm 0.08$ & \\
\hline & & & \\
\hline
\end{tabular}

CD: complete denture; PRD: partial removable denture; S-Significant $(p<$ 0.05).

Table 2. Mean maximum bite force values (Newton) and standard error of the right and left molar regions in the different analyzed groups (ANOVA).

\begin{tabular}{lccc}
\hline \multicolumn{1}{c}{ Groups and Regions } & N & $\begin{array}{c}\text { Means and } \\
\text { Standard Error }\end{array}$ & Significance \\
\hline Right Molar Region & & & S \\
Implants and Single Crowns & 10 & $254 \pm 31$ & \\
Upper CD and Lower Overdenture & 10 & $86 \pm 13$ & \\
Upper and Lower CD & 10 & $55 \pm 13$ & \\
Upper and Lower PRD & 10 & $135 \pm 20$ & S \\
Control & 10 & $350 \pm 54$ & \\
Left Molar Region & & & \\
Implants and Single Crowns & 10 & $224 \pm 25$ & \\
Upper CD and Lower Overdenture & 10 & $97 \pm 10$ & \\
Upper and Lower CD & 10 & $50 \pm 12$ \\
Upper and Lower PRD & 10 & $152 \pm 21$ & \\
Control & 10 & $388 \pm 80$ & \\
\hline
\end{tabular}

S: Significant $(p<0.01)$. 
Table 3. Correlation coefficients [r] between masticatory efficiency and maximum bite force indexes in the analyzed groups [Pearson's Bivariate correlation].

\begin{tabular}{cccccc}
\hline Clinical Condition & r-Force Right Molar & Sig & r-Force Left Molar & Sig & N \\
\hline Habitual Mastication & 0.447 & S & 0.394 & S & 50 \\
Mastication on the Right Side & 0.280 & S & 0.195 & 50 \\
Mastication on the Left Side & 0.418 & S & 0.264 & 50 \\
\hline
\end{tabular}

S: Significant $(p<0.05)$.

esthetical problems, thus reducing self-esteem. Edentulism is a chronic condition. Oral rehabilitations replace the missing teeth and aim at solving or minimizing those problems, recovering function, esthetics and mastication organ health so the individuals can eat and speak more easily and feel better about themselves [7]. These rehabilitations include conventional complete dentures, partial removable dentures, overdentures, bone-integrated implants and fixed dentures [8].

Some studies have shown that individuals rehabilitated with complete or partial dentures show lower masticatory efficiency indexes than those of individuals with a complete and healthy dentition, or rehabilitated with fixed dentures. Complete removable dentures, though having adequate retention and stability, are not capable of allowing individuals to have satisfactory mastication compared to people with natural dentition [9]. These results agree with those found in the present study which found higher masticatory efficiency indexes for individuals in the control group, dentate, and in the group rehabilitated with single-crowns over implants. The group rehabilitated with complete dentures showed lower masticatory efficiency indexes, $100 \%$ lower than dentate individuals [10].

According to Misch [11], fixed dentures exhibit better efficiency compared to removable dentures supported by soft tissue. This fact was verified in our study for both masticatory efficiency and force.

The treatment with dentures retained or supported by mandibular implants has shown considerable improvements on masticatory efficiency. When well indicated and executed, they may improve oral function, overcoming conventional complete dentures [8]. In the present study, the group rehabilitated with conventional complete upper dentures and lower overdenture inferior retained by two implants showed intermediate masticatory efficiency indexes, approximately $50 \%$ greater than those of the group rehabilitated with conventional complete dentures. These indexes were similar to those observed in the group rehabilitated with removable partial dentures. De Baat [12] stated that the most common disadvantages associated to using removable partial dentures are that it demands more oral hygiene control, as poor oral hygiene can harm the remaining dentition.
These disadvantages may be extreme in the elderly because they usually have a high amount of bacterial plaque, root caries and periodontal disease.

According to Berretin-Felix et al. [13] the type of dental treatment used has a direct relationship with masticatory efficiency, which is higher in individuals rehabilitated with tooth-supported and implant-supported dentures compared to individuals who use conventional complete dentures. In this study, the group of individuals rehabilitated with implants and single crowns showed high masticatory efficiency indexes, even higher than those observed in the control group. One possible explanation for this fact is the lack of periodontal ligaments, which reduced the proprioceptive capacity of the implants. The periodontal ligament has mechanorreceptors that send out information to the brain about the forces exerted by the teeth and these signals are involved in the neural control of mastication [14]. Its efficiency results in a smaller control of the bite force exerted [15].

Masticatory performance has been studied in individuals rehabilitated with conventional complete dentures and it has been found that they have only $25 \%$ of masticatory performance compared to dentate individuals. It has been proven that masticatory efficiency is significantly better in individuals who use overdentures over implants compared to individuals using conventional complete dentures [3]. These data are similar to those observed in the present study, in which individuals rehabilitated with upper complete denture and lower overdenture present better masticatory efficiency compared to the group of individuals rehabilitated only with conventional complete dentures, either in the clinical conditions of habitual mastication or mastication on only the right or left sides. The group of individuals using conventional complete dentures showed the worst masticatory performance among the evaluated groups.

Berretin-Felix et al. [13], evaluated the effects of lower implant-supported dentures on mastication and deglutition in completely edentulous elderly individuals. They verified that after the rehabilitation with implant-supported dentures, individuals showed significant improvement in the mastication and deglutition process of food. They also stated that other proposals for rehabilitation have been developed aiming at results hat would improve the mas- 
tication process considering that the difficulty in mastication was related to the instability of the dentures and the age of the individuals, thus, the implant-supported oral rehabilitations provided a masticatory performance similar to that observed in individuals with natural dentition, besides increasing bite force.

The masticatory efficiency may be defined as the capacity to grind a certain amount of food in a specific period of time. In the present study, beads were used to verify the masticatory efficiency indexes. In this method, the test material can be immediately evaluated and has stable physical properties. The advantage over the capsule is that its content is entirely preserved and there is no risk of being swallowed or dissolved by saliva. The laboratory processing is quick and effective, and permits to make a precise measurement of the patient's masticatory efficiency [16]. Previous studies have shown that this method is effective to determine the masticatory efficiency index $[6,16,17]$.

The masticatory force, an important factor for the growth of all facial bones, particularly the maxilla and mandible, depends on the periodontal proprioception. Rosa et al. [1], found that the mean bite force values of partially edentulous individuals were much smaller than those observed in dentate individuals, which shows the importance of oral prosthetic rehabilitation to restore the conditions of normality of the stomatognathic system, in terms of maximum bite force.

The highest mean maximum bite force values were found in the control group and in the group of individuals rehabilitated with implants and single crowns, followed by the group with removable partial dentures, the group with complete dentures and implant-retained mandibular overdentures and, finally, by the group of complete dentures.

The mean maximum bite force values in the control group were $350 \pm 54 \mathrm{~N}$ on the right side, and $388 \pm 80 \mathrm{~N}$ on the left. These values are similar to those found by other authors [4,5].

Rismanchian et al. [18], evaluated the maximum bite force in three groups of individuals: 1) individuals using conventional complete dentures with up to six months of use; 2) individuals using conventional complete dentures with ten or more years of use; and 3) individuals using upper complete dentures with lower implant-retained overdentures. The mean maximum bite force values in the three studied groups were respectively: $55.4 \pm 14.31$ $\mathrm{N} ; 68.74 \pm 20.59 \mathrm{~N}$ and $119.84 \pm 26.47 \mathrm{~N}$. These results are similar to those found in the present study, in which the mean values for the group of individuals rehabilitated with conventional complete dentures were $55 \pm 13 \mathrm{~N}$ on the right side and $50 \pm 12 \mathrm{~N}$ on the left side, and in the group of individuals rehabilitated with lower implantretained overdentures the means were $86 \pm 13 \mathrm{~N}$ on the right side, and $97 \pm 10 \mathrm{~N}$ on the left side. These values indicate that the rehabilitation with implants can be an important differential in improving the bite force in edentulous individuals, thus improving the masticatory function and the individuals' satisfactions [8].

According to Caloss et al. [19], the increased bite force and masticatory performance values in individuals who use conventional complete dentures and receive new implant-supported dentures may be explained by the greater stability of implant-retained dentures. They also stated that implant-supported dentures do not provide edentulous individuals the same functional level of totally dentate individuals, as the maximum bite force remains at least $20 \%$ smaller. In the present study, greater bite force values were found for the group of individuals rehabilitated with complete dentures and lower implantretained overdentures compared to the group of individuals with conventional complete dentures, though these values were much smaller than those registered in the control group.

\section{CONCLUSION}

In the data correlation analysis between masticatory efficiency and maximum molar bite force of the stomatognathic system, it was observed that the correlation between the data was positive for all the analyzed groups, in habitual mastication as well as in mastication on the right and left sides, evincing that increased bite force is directly related to the increase in masticatory efficiency. In the individual analysis of the proposed treatments, it was found that the correlation is much greater in habitual mastication. Therefore a correlation between maximal voluntary bite force and masticatory performance should be expected regardless of the proposed rehabilitating treatment. This study evinced that the group of individuals rehabilitated with implants and single crowns showed the greatest bite force and masticatory efficiency values compared to the other groups, the treatment using mandibular implant-retained overdentures improved function compared to using conventional complete dentures, and the treatment with implants was the one that provided the best rehabilitation of stomatognathic system functions.

\section{ACKNOWLEDGEMENTS}

The authors wish to thank FAPESP (2008/51409-8) for financially supporting this research.

\section{REFERENCES}

[1] Rosa, L.B., Semprini, M., Siéssere, S., Hallak, J.E.C., Pagnano, V.O. and Regalo, S.C.H. (2009) Correlation between bite force and electromyographic activity in dentate and partially edentulous individuals. Electromyography and clinical neurophysiology, 49, 291-297. 
[2] Van der Bilt, A. (2002) Human oral function: A review. Brazilian Journal of Oral Sciences, 1, 7-18.

[3] Pereira, L.J., Gavião, M.B., Bonjardim, L.R., Castelo, P.M. and Andrade Ada, S. (2006) Ultrasonography and electromyography of masticatory muscles in a group of adolescents with signs and symptoms of TMD. Journal of Clinical Pediatric Dentistry, 30, 314-319.

[4] Regalo, S.C.H., Santos, C.M., Vitti, M., Regalo, C.A., Vasconcelos, P.B., Mestriner-Junior, W., Semprini, M., Dias, F.J., Hallak, J.E. and Siéssere, S. (2008) Evaluation of molar and incisor bite force in indigenous compared with white population in Brazil. Archives of Oral Biology, 53, 282-286. doi:10.1016/j.archoralbio.2007.10.003

[5] Van der Bilt, A., Tekamp, A., Van der Glas, H. and Abbink, J. (2008) Bite force and electromyograpy during maximum unilateral and bilateral clenching. European Journal of Oral Sciences, 116, 217-222. doi:10.1111/j.1600-0722.2008.00531.X

[6] Felício, C.M., Couto, G.A., Ferreira, C.L. and Mestriner-Junior, W. (2008) Reliability of masticatory efficiency with beads and correlation with the muscle activity. Pro-Fono, 20, 225-230.

[7] Awad, M.A., Lund, J.P., Shapiro, S.H., Locker, D., Klemetti, E., Chehade, A., Savard, A. and Feine, J.S. (2003) Oral health status and treatment satisfaction with mandibular implant overdentures and conventional dentures: A randomized clinical trial in a senior population. International Journal of Prosthodontics, 16, 390-396.

[8] Feine, J.S. and Lund, J.P. (2006) Measuring chewing abiity in randomized controlled trials with edentulous populations wearing implant prostheses. Journal of Oral Rehabilitation, 33, 301-308. doi:10.1111/j.1365-2842.2006.01614.x

[9] Carlsson, G.E. (1984) Masticatory efficiency: The effect of age, the lost of teeth and prosthetic rehabilitation. International Dental Journal, 34, 93-97.

[10] Karkazis, H.C. (2002) EMG activity of the masseter muscle in implant supported overdenture wearers during chewing of hard and soft food. Journal of Oral Rehabilitation, 29, 986-991.
doi:10.1046/j.1365-2842.2002.00943.X

[11] Misch, C.E. (2007) Contemporary Implant Dentistry. 3rd Edition, Mosby, St. Louis.

[12] De Baat C. (2009) Elderly people and removable partial dentures. Ned Tijdschr Tandheelkd, 116, 665-668.

[13] Berretin-Felix, G., Nary Filho, H., Padovani, C.R., Trindade Junior, A.S. and Machado, W.M. (2008) Electromyographic evaluation of mastication and swallowing in elderly individuals with mandibular fixed implantsupported prostheses. Journal of Applied Oral Science, 16, 116-121. doi:10.1590/S1678-77572008000200007

[14] Johansson, A.S., Svensson, K.G. and Trulsson, M. (2006) Impaired masticatory behavior in subjects with reduced periodontal tissue support. Journal of Periodontology, 77, 1491-1497. doi:10.1902/jop.2006.050355

[15] Williams, W.N. and LaPointe, L.L. (1987) Vibratory stimulation of the masseter musculature and bite force discrimination. Journal of Craniomandibular Practice, 5, 25-31.

[16] Escudeiro, S.C., Freitas, O., Spadaro, A.C.C. and Mestriner-Junior, W. (2006) Development of a colorimetric system for evaluation of the masticatory efficiency. Brazilian Dental Journal, 17, 95-99. doi:10.1590/S0103-64402006000200002

[17] Farias Neto, A., Mestriner-Junior, W. and Carreiro, A.F. (2010) Masticatory efficiency in denture wearers with bilateral balanced occlusion and canine guidance. Brazilian Dental Journal, 21, 165-169. doi:10.1590/S0103-64402010000200013

[18] Rismanchian, M., Bajoghli, F., Mostajeran, Z., Fazel, A. and Eshkevari, P. (2009) Effect of implants on maximum bite force in edentulous patients. Journal of Oral Implantology, 35, 196-200. doi:10.1563/1548-1336-35.4.196

[19] Caloss, R., Al-Arab, M., Finn, R.A., Lonergan, O. and Throckmorton, G.S. (2010) Does long-term use of unstable dentures weaken jaw muscles? Journal of Oral Rehabilitation, 37, 256-261. doi:10.1111/j.1365-2842.2009.02046.x 